Tom Bode*, Kai Höltje, Sara Leal-Marin, Dr.-Ing. Marc Müller, Prof. Prof. h.c. Dr.-Ing. Birgit Glasmacher

\title{
Evaluation and Implementation of Biocompatible Methods for the Cross-linking of Plasma Proteins
}

\begin{abstract}
Autologous plasma proteins can be used to fabricate patient specific cardiovascular implants but need to be cross-linked to increase their mechanical strength and reduce water solubility. Glutaraldehyde is the state-of-the-art solution but its reaction products have been shown to be cytotoxic and pro-inflammatory. In this work, it has been shown, that cross-linking of plasma proteins with biocompatible alternatives to glutaraldehyde is possible. This was achieved by identifying four candidate substances (thrombin, transglutaminase, genipin, EDC) from current literature and investigating their ability to cross-link porcine plasma proteins in vitro. The degree of crosslinking was examined using calorimetric (DSC) and spectroscopic (FTIR, Raman) methods, mapping the influence of cross-linking on the denaturation temperature and primary amino-group content of the proteins. It could be shown that thrombin, genipin and EDC are able to cross-link plasma proteins to a satisfactory degree and thus represent useful alternatives to glutaraldehyde. Transglutaminase, on the other hand, could not sufficiently cross-link the plasma proteins and was therefore ruled out as an alternative.
\end{abstract}

Keywords: biocompatible crosslinking, blood plasma, EDC, proteins, genipin, thrombin, transglutaminase, cardiovascular implants, glutaraldehyde

https://doi.org/10.1515/cdbme-2021-2048

\section{Introduction}

Pathologically altered blood vessels pose a serious threat to the individuals health, especially in industrialised countries like

*Tom Bode: Institute for Multiphase Processes, Leibniz University Hannover, An der Universität 1, 30823 Garbsen, Germany, e-mail: t.bode@imp.uni-hannover.de

Kai Höltje, Sara Leal-Marin, Dr.-Ing. Marc Müller, Prof. Prof. h.c. Dr.-Ing. Birgit Glasmacher: Institute for Multiphase

Processes, Leibniz University Hannover, Hannover, Germany
Germany. Here, pathological changes of the cardiovascular system accounted for $12.83 \%$ of total deaths in 2018 [1]. In practice, the replacement of severely damaged or altered blood vessels with vascular prostheses often leads to a strong foreign body reaction of the organism. This is triggered, among other things, by the materials used in the prostheses. The final consequence of this incompatibility is failure of the prostheses or reduction of clinical success [2].

Prostheses made of autologous materials can prevent these reactions. Previous studies have shown that plasma proteins show high potential for use as cardiovascular implants [3]. Due to the water solubility of the proteins, as well as to increase the mechanical strength of the prosthesis, it is necessary to cross-link them. The structure and composition of the proteins must be preserved. State of the art is the use of glutaraldehyde for crosslinking, as shown in the successful cross-linking [4]. However, the products formed during the crosslinking reaction have been shown in several studies to have cytotoxic and pro-inflammatory properties. This increases the prospect of complications with the implants used [5-7]. It was therefore the purpose of this study to evaluate and implement different biocompatible crosslinking methods to be used for autologous cardiovascular implant fabrication at the Institute of Multiphase Processes.

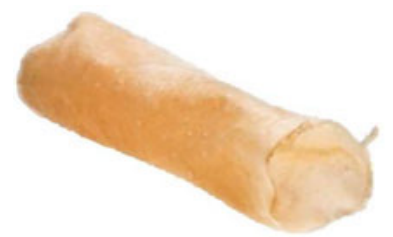

Figure 1: Vascular graft made from autologous blood proteins, cross-linked using glutaraldehyde vapor [Kühnhold, D. 2016].

In the course of a literature search and a utility analysis, four possible alternatives for crosslinking were selected. These are the enzymes transglutaminase (TG) and thrombin (Th) as well as the synthetic crosslinkers genipin (GP) and 1-Ethyl-3(3-dimethylaminopropyl)-carbodiimid (EDC) [4, 8-13]. High biocompatibility and low cytotoxicity of the additives are to be given the highest weighting. In addition, an evaluation was made according to the necessary reaction conditions for crosslinking. 
The selected crosslinkers were then tested in trials to determine their suitability for crosslinking plasma proteins. A comparative analysis of the crosslinking was performed using differential scanning calorimetry (DSC), Fourier transform infrared spectroscopy (FTIR) and Raman spectroscopy.

\section{Materials and Methods}

\subsection{Materials}

Porcine whole blood was provided by a local slaughterhouse and mixed with citrate $[3.13 \%(\mathrm{w} / \mathrm{v})$, trisodium citrate dihydrate; Sigma-Aldrich Chemie $\mathrm{GmbH}$ ] in a volume ratio of 1:10 (blood to citrate). The blood was then centrifuged in $50 \mathrm{ml}$ centrifugation tubes [Sarstedt $\mathrm{AG} \& \mathrm{Co}$ ] at an acceleration of $2561 \mathrm{G}$ (equivalent to $3500 \mathrm{rpm}$ ) for 10 minutes [Heraeus Megafuge $1 \mathrm{~S}-\mathrm{R}$; Thermo Fisher Scientific GmbH]. After centrifugation, the plasma was pipetted off.

In total, blood was processed on two different dates at intervals of several weeks. In each case, between 7 and 10 litres of blood from several pigs were centrifuged as quickly as possible after collection and stored under refrigeration. Using the plasma from the first batch, the genipin test series was set up. The plasma of the second batch was used to prepare the test series with EDC in ethanol as well as phosphate buffer saline (PBS), thrombin, transglutaminase and the reference samples.

EDC in the form of EDC-methiodide, bovine thrombin, genipin and calcium chloride dehydrate were purchased from Sigma-Aldrich GmbH. Transglutaminase was purchased from Würzteufel GmbH. Ethanol [96\% (v/v), denatured; Carl Roth $\mathrm{GmbH}]$ and a PBS solution ( $\mathrm{pH}$ 7.4) were used as solvents.

\subsection{Sample Preparation}

The parameters and compositions of the crosslinking solutions were chosen based on literature data. For this purpose, studies were selected which dealt with the crosslinking of proteins with the previously defined crosslinking reagents (genipin, transglutaminase, thrombin, EDC). Studies in which crosslinking was successfully achieved were filtered out and the parameters used were analysed. Based on these values, the parameters listed in Table 2.1 were chosen for further testing [4, 9, 13-19]. Before treatment, the plasma has to be mixed with calcium chloride. This serves to neutralize the citrate added as an anticoagulant. This is followed by an incubation period of 24 hours at a temperature of $37^{\circ} \mathrm{C} .15 \mathrm{ml}$ of plasma is used per sample.
Table 2.1: Overview of the parameters and solvents chosen for the crosslinking reactions to be performed.

\begin{tabular}{lll}
\hline Crosslinker & Content & Solvent \\
\hline GP & $25 \mathrm{mM}$ & EtOH \\
TG & $10 \mathrm{U} / \mathrm{ml}$ & PBS \\
Th & $20 \mathrm{U} / \mathrm{ml}$ & PBS \\
EDC & $30 \mathrm{mM}$ & EtOH/PBS \\
\hline
\end{tabular}

\subsection{Differential Scanning Calorimetry}

The influence of the reagents used on the properties of the samples compared to a reference made of untreated plasma allows conclusions to be drawn about the relative degree and quality of crosslinking. Stronger cross-linking leads to an increase in denaturation temperature which can be determined using DSC [8, 11, 13]. To perform the thermal analysis, sample crucibles containing 5 to $12 \mathrm{mg}$ of sample were prepared and placed individually in the DSC 204F1 from Netzsch. The device was controlled with the aid of the "DSC204" software. One cycle consisted of a heating process up to $140{ }^{\circ} \mathrm{C}$ with a heating rate of $5 \mathrm{~K} / \mathrm{min}$ and a subsequent isothermal holding time of $3 \mathrm{~min}$. For each material the experiment was performed five times. The denaturation temperatures were determined using the "Proteus Analysis" software. In the GP series, the maximum test temperature was $400{ }^{\circ} \mathrm{C}$ and the maximum heating rate $10 \mathrm{~K} / \mathrm{min}$. The maximum test temperature of the TG series was $200{ }^{\circ} \mathrm{C}$. The sample consisting of pure plasma was tested up to a maximum temperature of $160{ }^{\circ} \mathrm{C}$.

\subsection{Fourier-transform Infrared Spectroscopy}

Stronger cross-linking leads to a decrease in free primary $\varepsilon$-amino groups of amino acids. These properties are mapped with the selected analytical methods against a sample of unaltered plasma as a reference $[8,11,13]$. The spectra were recorded using an FTIR spectroscope from PerkinElmer Inc. [Spectrum 100, Perkin Elmer]. For each sample, spectra have been recorded in a wavenumber range from 600 to $4000 \mathrm{~cm}^{-1}$. Five samples per crosslinker were examined, with eight replicates for each acquisition. A background subtraction is performed after the measurement by means of a previously performed recording of the background radiation. Using the software "Spectragryph" the spectra were smoothed (SavitskyGolay filter, 3rd degree polynomial, interval 10 data points) and normalized to the highest peak. 
For a comparison of the spectra among each other, similar spectra of a test series were combined into one spectrum for each series [20].

\subsection{Raman-Spectroscopy}

For the recording of the Raman spectra a Raman spectrometer from the company Witec [alpha $300 \mathrm{RA}$ ] is used. The samples were excited with a laser (wavelength $532 \mathrm{~nm}$, power $12 \mathrm{~mW}$ ). For each crosslinker, five samples were analysed. Three images were taken per sample with a total acquisition time of 80 seconds over a wavenumber range from 0 to $3800 \mathrm{~cm}^{-1}$. Following the measurements, the spectra were smoothed with the software "Spectragryph" (Savitsky-Golay filter, 3rd degree polynomial, interval 10 data points), the background noise and interference due to cosmic rays were filtered out, and a background subtraction was performed. In addition, the spectra were normalized to the highest peak in each case. Similar spectra of the same crosslinker were merged for easier comparison [20].

\section{Results and Discussion}

\subsection{Sample Morphology}

After the incubation period, samples with genipin showed large solid structures of up to $2.5 \mathrm{~cm}$ in length at the bottom of the sample vessels. Similar observations could be made for samples with thrombin. Samples containing transglutaminase developed small spherical structures with diameters varying between 1 and $3 \mathrm{~mm}$. The addition of EDC lead to the formation of a finely dispersed white precipitate within in the samples.

\subsection{Protein Denaturation Temperature}

The resulting solids were separated from excess solvent and subjected to DSC analysis. As discussed above, a higher degree of cross-linking leads to a higher denaturation temperature by increasing the number of bonds between peptides and proteins. Here, samples with TG showed an increase in denaturation temperature compared to the reference from $75.5 \pm 0.7{ }^{\circ} \mathrm{C}$ to $143.0 \pm 4.2{ }^{\circ} \mathrm{C}$. This increase shows the strong cross-linking of proteins by TG compared to the other reagents used. Treatment of plasma samples with GP resulted in an increase to $110.1 \pm 5.4{ }^{\circ} \mathrm{C}$. In samples with EDC, the denaturation temperature decreased to $62.0^{\circ} \mathrm{C}$.
Samples with Th could not be analysed calorimetrically due to laboratory closures caused by the pandemic.

\subsection{Spectroscopic Analysis}

As previously discussed, spectroscopic methods were used to determine changes in the samples free primary $\varepsilon$-amino groups. In FTIR-Spectroscopy, samples with Th showed a strong decrease in primary amino groups compared to the plasma reference in all relevant wave number ranges, followed by EDC. The decrease was significantly lower for TG and GP. In this comparison, Th provides the highest relative degree of cross-linking. In Raman spectroscopy, TG showed the weakest signals for primary amines (1556 and $\left.1665 \mathrm{~cm}^{-1}\right)$ and the strongest for secondary ones $\left(3071 \mathrm{~cm}^{-1}\right)$ compared to the other cross-linkers. The degree of cross-linking is thus highest in comparison. Thrombin showed similarly strong signals for primary amines, but significantly lower for secondary ones. EDC showed the strongest peaks for primary amines in this study, suggesting the lowest degree of cross-linking. Samples with GP could not be analysed with this method due to fluorescence.

\section{Conclusion}

In the course of this study, it was shown that treatment of the plasma samples with genipin as well as thrombin leads to a clear cross-linking of the contained proteins. Genipin was able to provide for an optically well recognizable fusion of the proteins of the plasma. However, the content of primary amino groups did not exceed that of the samples cross-linked by thrombin. Optically, an almost complete, solid coalescence of the solution could be observed in the sample vessels with thrombin. These properties make it the most promising glutaraldehyde alternative.

Similarly, for samples with EDC, strong cross-linking of the proteins could be demonstrated spectroscopically. However, in an optical assessment, they did not exhibit cohesive solid protein assemblies. The significantly lower denaturation temperature can be attributed to the use of ethanol. This potentially denatures the proteins and thus ensures pre-damage and a reduction in cross-linking and crosslinkability. The small-scale crosslinking in TG samples could be due to too low a concentration of the crosslinker used or insufficient activity of the enzyme which resulted in an insufficiently high distribution and ability of the TG for crosslinking a large amount of plasma proteins in the samples. A similar problem could have influenced the EDC samples. 
The methods used in this work were applied for the first time at the Institute for Multiphase Processes. For this purpose, they were extended to provide reproducible information for the analysis of plasma proteins. The findings and formulated investigation methods of this work lay an important foundation for the further development of protein-based and crosslinked implants at the Institute for Multiphase Processes.

\section{Author Statement}

This project was funded by the Leibniz Young Investigator Grants of Leibniz Universität Hannover "Patient-specific implants from autologous blood donations". Authors state no conflict of interest. The research related to animal use complied with all the relevant national regulations, institutional for the care and use of animals.

\section{References}

[1] DESTATIS.: Die 10 häufigsten Todesfälle durch HerzKreislauf-Erkrankungen. 15.05.2020. https://www.destatis.de/DE/Themen/GesellschaftUmwelt/Gesundheit/Todesursachen/Tabellen/sterbefaell e-herz-kreislauf-erkrankungen-insgesamt.html. - Date of access: 13.04.2021.

[2] Müller, M.; Krolitzki, B.; Glasmacher, B.: Dynamic in vitro hemocompatibility testing - improving the signal to noise ration. In: Biomedical Engineering / Biomedizinische Technik 57 (2012), S. 549 - 552.

[3] Stegemann, J. P.; Kaszuba, Stephanie N.; Rowe, S. L.: Review: advances in vascular tissue engineering using protein-based biomaterials. In: Tissue engineering 13 (2007), Nr. 11, S. 2601-2613.

[4] Sell, S. A.; Francis, M. P.; Garg, K.; McClure, M. J.; Simpson, D. G.; Bowlin, G. L.: Cross-linking methods of electrospun fibrinogen scaffolds for tissue engineering applications. In: Biomedical materials (Bristol, England) 3 (2008), Nr. 4.

[5] Gough, J. E.; Scothcford, C. A.; Downes, S.: Cytotoxicity of glutaraldehyde crosslinked collagen/poly(vinyl alcohol) films is by the mechanism of apoptosis. In: Journal of biomedical materials research 61 (2002), Nr. 1, S. 121-130.

[6] Huang-Lee, L. L. H.; Cheung, D. T.; Nimni, M. E.: Biochemical changes and cytotoxicity associated with the degradation of polymeric glutaraldehyde derived crosslinks. In: Journal of biomedical materials research 24 (1990), Nr. 9, S. 1185-1201.

[7] Speer, D. P.; Chvapil, M.; Eskelson, C. D.; Ulreich, J.: Biological effects of residual glutaraldehyde in glutaraldehyde-tanned collagen biomaterials. In: Journal of biomedical materials research 14 (1980), Nr. 6, S. 753-764.

[8] Sung, H. W.; Liang, I.-L.; Chen, C.-N.; Huang, R.-N.; Liang, H.-F.: Stability of a biological tissue fixed with a naturally occurring crosslinking agent (genipin). In: Journal of biomedical materials research. Part A 55 (2001), S. 538-546.
[9] Schek, R. M.; Michalek, A. J.; Iatridis, J. C.: Genipincrosslinked Fibrin hydrogels as a potential adhesive to augment intervetrebal disc annulus repair. In: European Cells \& Materials 21 (2011), S. 373-383.

[10] Aper, T.; Wilhelmi, M.; Gebhardt, C.; Hoeffler, K.; Benecke, N.; Hilfiker, A.; Haverich, A.: Novel method for the generation of tissue-engineered vascular grafts based on a highly compacted fibrin matrix. In: Acta biomaterialia 29 (2016), S. 21-32.

[11] Perumcherry, S. R.; Chennazhi, K. P..; Nair, S. V.; Menon, D.; Afeesh, R.: A Novel Method for the Fabrication of Fibrin-Based Electrospun Nanofibrous Scaffold for Tissue-Engineering Applications. In: Tissue Engineering Part C: Methods 17 (2011), Nr. 11, S. 1121-1130.

[12] Li, P.-S.; Lee, I-L.; Yu, W.-L.; Sun, J.-S.; Jane, W.-N.; Shen, H.-H.: A novel albumin-based tissue scaffold for autogenic tissue engineering applications. In: Scientific reports 4 (2014), S. 5600.

[13] Zhu, K.; Slusarewicz, P.; Hedman, T.: Thermal Analysis Reveals Differential Effects of Various Crosslinkers on Bovine Annulus Fibrosis. In: Journal of orthopaedic research: official publication of the Orthopaedic Research Society 29 (2011), Nr. 1, S. 8-13.

[14] Ahmad, Z.; Shepherd, J. H.; Shepherd, D. V.; Ghose, S.; Kew, S. J.; Ameron, R. E.; Best, S. M.; Brooks, Roger A.; Wardale, J.; Rushton, Neil: Effect of 1-ethyl-3(3-dimethylaminopropyl) carbodiimide and $\mathrm{N}$ hydroxysuccinimide concentrations on the mechanical and biological characteristics of cross-linked collagen fibres for tendon repair. In: Regenerative biomaterials 2 (2015), Nr. 2, S. 77-85.

[15] Dimida, S.; Barca, A.; Cancelli, N.; de Benedictis, V.; Raucci, M. G.; Demitri, C.: Effects of Genipin Concentration on Cross-Linked Chitosan Scaffolds for Bone Tissue Engineering: Structural Characterization and Evidence of Biocompatibility Features. In: International Journal of Polymer Science 2017 (2017), Nr. 360, S. 1-8.

[16] Everaerts, F.; Torrianni, M.; Hendriks, M.; Feijen, J.: Biomechanical properties of carbodiimide crosslinked collagen: influence of the formation of ester crosslinks. In: Journal of biomedical materials research. Part A 85 (2008), Nr. 2, S. 547-555.

[17] Savoca, M. P.; Tonoli, E.; Atobatele, A. G.; Verderio, E. A. M.: Biocatalysis by Transglutaminases: A Review of Biotechnological Applications. In: Micromachines 9 (2018), Nr. 11, S. 1-23.

[18] Sung, H.-W.; Chang, W.-H.; Ma, C.-Y.; Lee, M.-H.: Crosslinking of biological tissues using genipin and/or carbodiimide. In: Journal of biomedical materials research. Part A 64 (2003), Nr. 3, S. 427-438.

[19] Swartz, D. D.; Russell, J. A.; Andreadis, S. T.: Engineering of fibrin-based functional and implantable small-diameter blood vessels. In: American journal of physiology. Heart and circulatory physiology 288 (2005), Nr. 3, S. H1451- 60.

[20] Menges, F.: Spectragryph: Optical Spectroscopy Software. 2020. - URL http://www.effemm2.de/spectragryph/. - Date of Access: 28.03.2020 